\title{
Targeting expression of expanded polyglutamine proteins to the endoplasmic reticulum or mitochondria prevents their aggregation Erwann Rousseau ${ }^{1}$, Benjamin Dehay ${ }^{1}$, Léa Ben-Haïem², Yvon Trottier², Michel Morange ${ }^{1}$ and Anne Bertolotti*1
}

\author{
Address: ${ }^{1}$ Laboratoire de Génétique Moléculaire, CNRS UMR8541, Ecole Normale Supérieure, 46 rue d'Ulm, 75230 Paris Cedex 05, France and \\ 2Institut de Génétique et de Biologie Moléculaire et Cellulaire, INSERM-CNRS-Université Louis Pasteur, BP 10142, 67404 Illkirch Cedex, France \\ * Corresponding author
}

from The 4th Recombinant Protein Production Meeting: a comparative view on host physiology Barcelona, Spain. 2I-23 September 2006

Published: 10 October 2006

Microbial Cell Factories 2006, 5(Suppl I):S7 doi:I0.I I86/I475-2859-5-SI-S7

(c) 2006 Rousseau et al; licensee BioMed Central Ltd.

Aggregation of misfolded proteins is a characteristic of several neurodegenerative diseases. The huntingtin amino-terminal fragment with extended polyglutamine repeat forms aggregates closely associated with chaperones both in the cytoplasm and the nucleus. As each cellular compartment contains distinct chaperones and because the molecular mechanisms controlling polyglutamine aggregation are largely unknown, we decided to investigate the influence of different cellular environments on the aggregation of this pathological protein. We found that aggregation of a protein containing a polyglutamine stretch of pathological length is abolished when its expression is targeted to the endoplasmic reticulum. Once retrogradely transported outside of the endoplasmic reticulum, the aggregation-prone polyglutamine containing protein recovers its ability to aggregate. When expressed in the mitochondria, a protein containing 73 glutamines is entirely soluble while the nucleo-cytosolic equivalent has an extremely high tendency to aggregate. Our data imply that polyglutamine aggregation is a property restricted to the nucleo-cytosolic compartment and suggest the existence of compartment-specific co-factors promoting or preventing aggregation of pathological proteins. Implications for recombinant proteins production will be discussed. 